\title{
The Interplay between Genes and Psychosocial Home Environment on Physical Activity
}

\author{
Sari Aaltonen ${ }^{1,2}$, Jaakko Kaprio ${ }^{1,3}$, Urho M. Kujala ${ }^{4}$, Lea Pulkkinen ${ }^{5}$, Richard J. Rose ${ }^{6}$, and \\ Karri Silventoinen ${ }^{2}$
}

${ }^{1}$ Institute for Molecular Medicine (FIMM), University of Helsinki, Helsinki, Finland ${ }^{2}$ Department of Social Research, University of Helsinki, Helsinki, Finland ${ }^{3}$ Department of Public Health, University of Helsinki, Helsinki, Finland ${ }^{4}$ Faculty of Sport and Health Sciences, University of Jyvaskyla, Jyväskylä, Finland ${ }^{5}$ Department of Psychology, University of Jyvaskyla, Jyväskylä, Finland ${ }^{6}$ Department of Psychological and Brain Sciences, Indiana University, Bloomington, IN

\section{Abstract}

Introduction-Genetic factors contribute to individual differences in physical activity, but it remains uncertain whether the magnitude of the genetic effects is modified by variations in home environments. We aimed to examine to what extent the psychosocial home environment in childhood and adolescence modifies the genetic influences on leisure-time physical activity in young adulthood.

Methods-Participants were Finnish twins $(\mathrm{N}=3,305)$ who reported their leisure-time physical activity at age 24 . The psychosocial home environment was assessed by twins at ages 12, 14 and 17 , as well as by their parents when the twins were age 12 . Gene-environment interaction modeling was performed with OpenMx software.

Results-Parental ratings of positive home atmosphere as well as the twins' ratings of both positive home atmosphere at age 14 and lower relational tensions at ages 12 and 14 predicted higher leisure-time physical activity levels in young adulthood (regression coefficients $0.33-0.64$ ). Parental perceptions as well as the twins' perceptions of positive home atmosphere at ages 14 and 17 increased the additive genetic variation (moderation effects $0.60,95 \%$ CI $0.26-1.05$; $0.55,95 \%$ CI $0.29-0.80$ and $0.52,95 \%$ CI $0.19-0.87$, respectively). The twins' ratings of positive home atmosphere at age 12 and lower relational tensions at ages 12 and 14 increased the unique

Corresponding author: Sari Aaltonen Ph.D., Institute for Molecular Medicine (FIMM), University of Helsinki, P.O. Box 20 (Tukholmankatu 8 B), FIN-00014 University of Helsinki, Helsinki, Finland. Tel: +358 504480025 Fax: +358 919127600 sari.s.aaltonen@helsinki.fi.

\section{AUTHOR CONTRIBUTIONS}

J.K., L.P. and R.J.R. conceived, designed and contributed to the data collection of the FinnTwin12 cohort. S.A., J.K. and K.S. designed the present study. S.A. and K.S. conducted the statistical analyses. S.A. drafted the manuscript and R.J.R., U.M.K., L.P., J.K. and K.S. critically revised and edited the manuscript. All authors read and approved the final manuscript.

\section{CONFLICTS OF INTEREST}

The authors declare no conflict of interest. The results of the present study do not constitute endorsement by the American College of Sports Medicine.

The authors state that the results of the study are presented clearly, honestly, and without fabrication, falsification, or inappropriate data manipulation. 
environmental variation of their subsequent physical activity (moderation effects $0.46,95 \% \mathrm{CI}$ $0.19-0.60 ; 0.48,95 \%$ CI $0.29-0.64$ and $0.85,95 \%$ CI $0.12-0.95$, respectively).

Conclusions-A psychosocial home environment that is warm and supportive in childhood and adolescence not only increases the mean level of subsequent leisure-time physical activity in young adulthood, but also modifies the genetic and environmental variances in leisure-time physical activity.

\section{Keywords}

Adolescence; Childhood; Exercise; Home atmosphere; Longitudinal; Twins

Numerous twin and family studies have indicated that both genetic and environmental factors contribute to individual differences in physical activity (1-4). To date, the proportion of the variance in physical activity attributable to genetic effects has been found to range from $27 \%$ to $84 \%$ in twin and family studies (1-6). Two of these studies, one from Finland (6) and the other from the Netherlands (4), have longitudinally tracked genetic and environmental influences in childhood, adolescence and young adulthood confirming that the nature of genetic and environmental influences affecting physical activity is not stable, (i.e., genetic and environmental influences are age- and population-specific). The role of environmental factors shared by co-twins, such as the home environment, has been suggested by both of these studies, but in the Dutch study shared environmental effects were very strong in early childhood. Due to the small effect of genetic and large effect of shared environmental factors in early childhood, the Dutch study suggested that the home environment can affect a child's physical activity behavior along with genetic influences.

Many non-genetic studies utilizing direct measures of the home environment have also suggested that the home environment affects physical activity behavior (7). Importantly, in addition to the physical home environment $(8,9)$, socioeconomic position of a family $(10$, 11 ) and built environment $(12,13)$, several reviews have also found that many psychosocial factors are involved in an individual's level of physical activity $(7,11,14-18)$. These reviews of dozens of studies published since 1970 have shown strong evidence that parental support is positively associated with an adolescent's physical activity level. Recently, an Australian study of families with younger (5-6 years old) and older children (10-12 years old) examined the longitudinal relationship between a family's physical activity environment (i.e., role modeling, family participation, support and reinforcement related to physical activity) and future physical activity behavior (19). The researchers followed the families over five years and found that there was a significant association between baseline home environment and subsequent moderate-to-vigorous physical activity among both younger and older children, even though the relationship was weak in magnitude. Another Australian study also focused on the causal effects of the home environment among children who were ages 10 to 12 at baseline (20). Interestingly, this study found that when different home environments were compared, the home environment including parental role modeling, social support, as well as rules and restrictions was more important than neighborhood physical activity environment factors in influencing the adolescents' physical activity behavior over five years. The importance of parental support was also emphasized by a study 
of young American girls, which showed that parental support for girls at age 9 was a significant predictor for the girls' physical activity behavior at age 11 (21).

The studies reviewed above suggest that sibling resemblance in the regular involvement in physical activity reflects a sharing of both the genetic background and home environment. However, the potential interactions between the genetic factors and home environment should also be studied when trying to explain how individual differences in physical activity behavior arise. Dzewaltowski et al. (2008) (22) showed that social bonding within a family moderated the relationship between the levels of the parents' and an adolescent's physical activity, which may suggest that the genetics of physical activity interact with the psychosocial home environment. However, to the best of our knowledge, no previous study has investigated whether the psychosocial home environment in childhood and adolescence can modify the genetic variance in physical activity, even though it is important to know whether the home environment can enhance or reduce the potential genetic propensity for physical activity. It is possible that due to genetic factors some children are more prone to the effects of the home environment than others, and this may have long-term effects on physical activity behavior.

Our aim was to examine the extent to which the psychosocial home environment in childhood and adolescence modifies the effects of genetic factors on leisure-time physical activity in young adulthood in Finnish twins. We chose to focus on leisure-time physical activity, because it may better express voluntary behavior and show greater variation by reflecting the wishes and/or inherent abilities of the twins than their daily total physical activity level. In addition, we examine whether the psychosocial home environment in childhood and adolescence modifies the effects of unique environmental factors on leisuretime physical activity in young adulthood. There are previous studies that have demonstrated the existence of clear gene-environment interaction between the psychosocial home environment and several factors, such as psychological traits (23), smoking behavior (24) and alcohol use (25). This suggests that the psychosocial home environment may modify the manifestation of the genetic potential related to human behavior. We speculate that it is also the case when studying leisure-time physical activity.

\section{METHODS PARTICIPANTS}

The participants were drawn from the longitudinal FinnTwin12 study focusing on health and behavior in Finnish twins born between 1983 and 1987, as well as their families (26). The twins were initially identified from the Central Population Registry of Finland, and the relevant data for the study were collected through mailed questionnaires. In the first phase of data collection, both the twins and their parents completed questionnaires when the twins were 11-12 years old. Subsequently, the twins were surveyed again at the mean ages of 14 (range 13.9-14.9), 17.6 (range 17.2-19.5) and 24.2 (range 20.5-27.5) (27). The response rates have been high in each wave of data collection, ranging from $73 \%$ to $90 \%$ (26).

There were 3,305 twin individuals who reported their level of leisure-time physical activity in young adulthood. Of these, 3,129 (57\% females) had reported on their home atmosphere 
at age 12, 2,997 (57\% females) at age 14, and 2,864 (58\% females) at age 17, while 3,137 (57\% females) reported on relational tensions at age 12, 3,012 (57\% females) at age 14, and 2,864 (58\% females) at age 17. Parental assessment of the home atmosphere was available for 3,043 twin individuals, and on relational tensions for 3,052 twin individuals who had reported their leisure-time physical activity at age 24 . The number of complete twin pairs with full data available for each zygosity group across the ages 12,14 and 17 were as follows: monozygotic (MZ) male pairs: $n=170-184$, MZ female pairs: $n=264-282$, dizygotic (DZ) male pairs: $\mathrm{n}=151-162$, DZ female pairs: $\mathrm{n}=217-225$, and DZ opposite sex pairs: $n=323-359$.

\section{ASSESSMENT OF LEISURE-TIME PHYSICAL ACTIVITY}

Leisure-time physical activity behavior was assessed at the latest wave of the study, when the twins had a mean age of 24 . The assessment of physical activity level was based on a series of structured questions on all types of leisure-time physical activity (i.e., monthly frequency, mean duration, and mean intensity of leisure-time physical activity sessions). The overall activity level was assessed as leisure-time metabolic equivalent units (MET index), which were created by assigning a multiple of the resting metabolic rate (MET score) to each activity, and by calculating the product of the activity, defined as intensity $\times$ duration $\times$ frequency. The MET indices were expressed as the sum score of leisure-time MET hours/ day. The MET values for leisure-time physical activity intensity were 4 for intensity corresponding to walking, 6 for intensity corresponding to vigorous walking to jogging, 10 for intensity corresponding to jogging, and 13 for intensity corresponding to running. Previous studies have shown moderate to high correlations between the leisure-time physical activity questions used in the present study and physical activity data obtained by interview $(\mathrm{r}=0.56, \mathrm{p}<0.001)(28)$ or by a detailed assessment of 12-month leisure-time physical activity history $(r=0.73, \mathrm{p}<0.001)(29)$.

\section{ASSESSMENT OF THE PSYCHOSOCIAL HOME ENVIRONMENT}

The psychosocial home environment was independently assessed by mothers and fathers, as well as by both co-twins when the twins were age 12 by using an 8 -item questionnaire. Twins later also reported their subjective view of their psychosocial home environment at ages 14 and 17. The following items were used in the questionnaire: 1) warm and caring, 2) creative and supportive, 3) trusting and understanding, 4) open, 5) hard discipline, 6) unjust, 7) argumentative, and 8) indifferent. A 5-point scale was used with options ranging from "strongly disagree" to "strongly agree". The first four items describe the home atmosphere, whereas the remaining items represent relational tensions between children and parents. We conducted an unrotated factor analysis using Stata software, version 14.1 (StataCorp, College Station, TX, USA), and found that one factor at every time-point explained the majority of the variation for the home atmosphere (62\% for twin ratings at age $12,70 \%$ for twin ratings at age $14,70 \%$ for twin ratings at age 17 and $62 \%$ for parental ratings) and the other factor for relational tensions (61\% for twin ratings at age $12,44 \%$ for twin ratings at age $14,61 \%$ for twin ratings at age 17 and $56 \%$ for parental ratings), whereas eigenvalues for the remaining factors were low (ranging 0.50 to 0.97 ), indicating only two relevant factors. Item number 5 (hard discipline) did not fit well on either of the factor scores and was therefore excluded from these analyses. Thus, the two created factor scores were used in 
the analyses and scaled in such a way that high scores indicate a positive home atmosphere and low relational tensions between children and parents.

\section{STATISTICAL METHODS}

We began the analyses by calculating the descriptive statistics for the psychosocial home environment and leisure-time physical activity. In these analyses, twins were analyzed as individuals, meaning that the observations and their error terms between members of a pair may be correlated, and, therefore, we adjusted the analyses for twin clustering (30) and used the Wald test for equality of means to derive the proper p-values and confidence intervals (CI). Furthermore, we calculated correlations between the psychosocial home environment and leisure-time physical activity variables by using polychoric correlations to estimate the associations between two normally distributed, continuous, latent variables from two observed ordinal variables. Again, we adjusted the analyses for twin clustering (31). All analyses were conducted using Stata, version 14.1 and the significance level was set at $\mathrm{p}<0.05$.

Data were further analyzed by using the quantitative genetic modeling of twin data based on the different genetic relatedness of $\mathrm{MZ}$ and $\mathrm{DZ}$ co-twins (31). The variance of leisure-time physical activity behavior can consist of four sources of variation: additive genetic variation (A), dominant genetic variation (D), common environmental variation (C), and unique environmental variation (E) (32). Measurement error is included in the unique environmental variation. MZ co-twins share approximately all of their genetic material (the correlation is 1.0 for additive and dominant genetic influences), DZ co-twins share, on average, $50 \%$ of their segregating genes (the correlation is 0.5 for additive and 0.25 for dominant genetic influences). Common environmental effects refer to all environmental influences that make members of a twin pair alike, so by definition they correlate 1.0 in both $\mathrm{MZ}$ and DZ twins. Unique environmental effects denote all environmental influences that make members of a twin pair not alike, and, thus, are uncorrelated in both MZ and DZ twins. Since all twins who participated in this study were reared together, we were unable to simultaneously estimate dominant genetic and common environmental influences.

Genetic modeling began by computing a univariate model (31). The findings of our previous study of genetic and environmental influences on longitudinal changes in leisure-time physical activity examining the same data-set used in the present study suggested that the full ACE -model would act as a reasonable starting point for genetic modeling (6). First, we determined whether common environmental factors are present to explain the variation in twins' leisure-time physical activity behavior at age 24 by comparing the full ACE -model to the AE -model; no difference between models indicates that the simpler model describes the data as adequately as the more complex model, and unnecessary parameters can consequently be eliminated. The comparison of models showed that common environmental components could be dropped from the model, because dropping the common environmental path coefficient did not lead to a significant deterioration of model fit ( $\mathrm{p}=0.58)$. Thus, the best fitting model, in which additive genetic and unique environmental components explain the variance and covariance of leisure-time physical activity was used in the gene-interaction modeling. 
Subsequently, we tested whether sex-specific genetic factors were related to leisure-time physical activity behavior by analyzing whether the genetic correlations for opposite-sex twins could be constrained to 0.5 . The analysis revealed no evidence for a sex-specific genetic effect ( $\mathrm{p}=0.66)$, indicating that the same genetic components appear to affect leisuretime physical activity in men and women. We also tested whether there were differences in genetic and environmental variances between men and women in leisure-time physical activity behavior in young adulthood, but found no significant differences in either absolute genetic and environmental variances $(\mathrm{p}=0.60)$ or relative genetic and environmental variances $(\mathrm{p}=0.66)$.

The hypothesis that the psychosocial home environment in childhood and adolescence interacts with the genetic effects on the twins' leisure-time physical activity in young adulthood was assessed with gene-environment interaction models (33). These models test whether the genetic and environmental effects differ between different levels of measured environmental exposures. In practice, we tested whether the genetic and environmental variances of leisure-time physical activity change as a function of the psychosocial home environment. Figure 1 illustrates the gene-environment interactions. In the figure, the moderator factor (psychosocial home environment) is denoted as $\mathrm{M}$. The moderator factor can affect the mean value of leisure-time physical activity $\left(\beta_{M}\right)$, as well as modify the effects of genetic $\left(\beta_{\mathrm{A}}\right)$ and unique environmental factors $\left(\beta_{\mathrm{E}}\right)$ on leisure-time physical activity. This means that the psychosocial home environment can affect both the mean value of leisuretime physical activity as well as genetic and environmental variances. The mean value of leisure-time physical activity is modeled as a mean moderation effect, which takes both gene-environment correlations and a causal effect of the psychosocial home environment on leisure-time physical activity into account.

Genetic modeling was carried out with OpenMx (version 2.0.1) software, which is a package for extended structural equation modeling on the R statistical platform (34). We analyzed data from men and women together, not only due to the similar sex-specific genetic effects and similar genetic and environmental variances found in men and women, but also due to statistical power requirements, because the detection of significant gene-environment interactions requires a large sample size. Further, we used both same-sex and opposite-sex twin pairs in the analyses, and the analyses were conducted using the raw data option, allowing for the inclusion of data from a single twin in the absence of their co-twin. Unstandardized parameter estimates were favored in order to examine an absolute change rather than proportional change. Statistically significant results were primarily judged by 95\% CIs, but we also calculated p-values for results that were initially shown to be significant based on $95 \%$ CIs. To be able to counteract the problem of multiple comparisons, Bonferroni multiple testing correction was applied to adjust the significance threshold ( $a$ / $\mathrm{n}=0.05 / 4, \mathrm{n}$ represents the number of tests performed in home atmosphere and relational tensions analyses), meaning results are significant at the $\mathrm{p}=0.012$ level.

\section{ETHICS OF THE STUDY}

The ethics committees of the Department of Public Health at the University of Helsinki (Helsinki, Finland) and the Helsinki University Central Hospital District (Helsinki, Finland), 
along with the Institutional Review Board of Indiana University (Bloomington, IN, USA) approved the FinnTwin 12 study protocol. The parents of the participating twins (at baseline) or twins themselves as young adults provided written informed consent for participation in the study.

\section{RESULTS}

Means and standard deviations of the home atmosphere and relational tensions, measured in childhood and adolescence, as well as leisure-time physical activity assessed in young adulthood are presented by sex and zygosity in Table 1. We assessed mean differences between sex and zygosity groups and found several differences in the home atmosphere and relational tensions variables that reached statistical significance ( $\mathrm{p}$-values from $<0.001$ to 0.04). Interestingly, $M Z$ twins almost systematically reported slightly higher values for a positive home atmosphere and lower relational tensions than DZ twins. However, many of these differences were small in magnitude and became statistically significant because of the large sample size. Parental ratings of the relational tensions when the twins were age $12 \mathrm{did}$ not differ between sexes or zygosities $\left(p_{\mathrm{sex}}=0.36\right.$ and $\left.p_{\text {zygosity }}=0.65\right)$. In terms of leisure-time physical activity, men were more active than women $\left(p_{\text {sex }}=0.009\right)$, but no differences by zygosity were found $\left(p_{\text {zygosity }}=0.22\right)$.

The twins' assessments of their home atmosphere and relational tensions in childhood and adolescence were positively correlated with each other across survey waves (Table 2); polychoric correlations ranged from 0.12 to 0.62 . Positive correlations were also found between the twins' and parents' assessments of the psychosocial home environment; these were moderate in size (polychoric correlations from 0.21 to 0.30 ) indicating some differences in the subjective perceptions of the home atmosphere and relational tensions between the generations. Most importantly, the twins' assessments of their psychosocial home environment were positively associated with leisure-time physical activity behavior in young adulthood (from 0.04 to 0.10 ). Parental assessments of the home atmosphere were more weakly correlated with the twins' subsequent leisure-time physical activity behavior $(\mathrm{r}=0.04)$, whereas no association was found between parental ratings of relational tensions and the twins' leisure-time physical activity in young adulthood $(\mathrm{r}=-0.0001)$.

The heritability (i.e., the proportion of total variance explained by genetic variance) for the leisure-time physical activity at age 24 was $61 \%$ (95\% CI 56-66\%), and the remaining proportion of the variance explained by unique environmental influences was 39\% (95\% CI 34-44\%). In the gene-interaction modeling, we confirmed that the twins' ratings of a positive home atmosphere at age 14, as well as their ratings of lower relational tensions at ages 12 and 14, predicted higher leisure-time physical activity at age 24. Regression coefficients for the moderation of the mean leisure-time physical activity by psychosocial home environment variables $\left(\beta_{\mathrm{M}} \mathrm{M}\right)$ ranged from $0.36(\mathrm{p}<0.001)$ to $0.64(\mathrm{p}<0.001)$ (Table 3$)$. There was a similar trend of positive association between parental ratings of the home atmosphere and the twins' leisure-time physical activity in young adulthood (regression coefficient $0.33(\mathrm{p}=0.004))$. 
Further analyses of the modification effects of the psychosocial home environment on genetic and unique environmental influences showed that the psychosocial home environment in mid- and late adolescence modified the additive genetic variation of the twins' leisure-time physical activity in young adulthood. Higher levels of a positive home atmosphere as rated by twins at ages 14 and 17, as well as parents, statistically significantly increased the additive genetic variation of the twins' leisure-time physical activity in young adulthood (moderation effects 0.60 (95\% CI 0.26-1.05), p <0.001; 0.52 (95\% CI 0.19-0.87), $\mathrm{p}=0.001$ and 0.55 (95\% CI 0.29-0.80), $\mathrm{p}<0.001$, respectively) (Table 3). Moreover, we found that a positive psychosocial home environment in childhood and mid-adolescence increased the unique environmental variation of the twins' future leisure-time physical activity; the variance moderation effects were statistically significant for home atmosphere at age 12 (moderation effect 0.46 (95\% CI 0.19-0.60), $\mathrm{p}=0.001$ ) and relational tensions at ages 12 (moderation effect 0.48 (95\% CI 0.29-0.64), $\mathrm{p}<0.001$ ) and 14 (moderation effect 0.85 (95\% CI 0.12-0.95), $\mathrm{p}=0.006$ ) (Table 3).

\section{DISCUSSION}

In this study, we focused on the effect of the childhood and adolescence psychosocial home environment on the mean level of leisure-time physical activity in young adulthood as well as its potential moderation effects on the genetic and environmental variances in that physical activity. Subjective assessments of a positive home atmosphere in mid-adolescence, as well as lower relational tensions between parents and children in childhood and midadolescence, increased the twins' mean level of leisure-time physical activity as young adults. Parental ratings of the home atmosphere when the twins were 12 years old had a similar increasing effect on the twins' level of leisure-time physical activity in young adulthood. Gene-environment interaction analyses revealed that the subjective ratings of having a psychosocial home environment that is warm and supportive in childhood and midadolescence increased both the genetic variance and the unique environmental variance in the twins' leisure-time physical activity in young adulthood. The home atmosphere experienced in late adolescence modified only the genetic influences on leisure-time physical activity. This may suggest the greater importance of the childhood psychosocial home environment as a modifying factor of the additive genetic and environmental influences on future physical activity behavior.

Our results are consistent with recent reviews confirming that the psychosocial home environment is involved in the level of physical activity $(7,11,14-18)$. The present results support the idea that there may be a potential long-term and predictive association between the psychosocial home environment in childhood and adolescence and physical activity behavior in later life (19, 21). Davison et al. (2006) (21) showed, in their two-year follow-up study, that parental support for young girls can lead to increased physical activity, and similarly, Cleland et al. (2011) (19) found a positive relationship between the home environment and subsequent moderate-to-vigorous physical activity after five years.

In our study, the follow-up period was longer than in the previously mentioned studies (19, 21), showing that the effect of the psychosocial home environment can last years, even across the transition from childhood to young adulthood. In the previous studies, only 
parents self-reported their view of the psychosocial home environment, while in our study we were able to take both the twins' and parents' perceptions into account. Our study design produced results consistent with previous evidence by showing that the twin and parental ratings of the home atmosphere in childhood and adolescence were only weakly correlated with the twins' leisure-time physical activity behavior. This was found even though the twin and parental ratings of the home atmosphere in childhood and adolescence were all only moderately, at most, correlated with the twins' leisure-time physical activity. These weak associations are likely explained by the fact that the influence of the childhood and adolescence home environment decreases when children grow up and other environmental factors involved in leisure-time physical activity behavior than those related to the childhood home environment appear.

We found that the psychosocial home environment in childhood and adolescence modified the genetic and environmental influences of leisure-time physical activity in young adulthood. As far as we know, this is the first study to explain these kinds of modifying roles, although there is a previous study that suggested moderating effects of the home environment on an offspring's physical activity behavior; a positive moderating effect of social bonds in families was revealed by Dzewaltowski et al. (2008) (22). Our results confirm this previous evidence by suggesting that if you believe your parents are emotionally warm, supportive and encouraging in your family interactions in childhood or mid-adolescence, it might enhance your genetic potential for voluntary physical activity, as well as enhance the utilization of your living environment to increase your physical activity behavior in young adulthood, whereas the same parental interactions in late adolescence may be less likely to lead to the same results. Thus, a positive psychosocial home environment is more likely to support a child's tendency to be physically active even in later life. It is important to note that the study by Dzewaltowski et al. (22) was a cross-sectional design that revealed concurrent associations between the factors, while the design we used was longitudinal, suggesting that the effect is also long-term and may potentially be causal.

In our study, the twins' subjective ratings of their psychosocial home environment were assessed in childhood and adolescence, not at the same time as the assessments of leisuretime physical activity in young adulthood. A disadvantage of this approach is that the physical activity level of the twins in childhood and adolescence may itself have affected the psychosocial home environment and parental attitudes toward their children. Thus, the measured association between the psychosocial home environment and physical activity behavior may have been biased by not taking this baseline physical activity behavior into account. However, an advantage of this approach is that it helps to prevent potential recall bias with regard to the childhood and adolescence home environment. Interestingly, it seemed that the children's subjective perception of their psychosocial home environment was more important than what the parents perceived, because the parents' subjective perceptions of the same home environment did not predict their offspring's future physical activity behavior as strongly as the offspring's own perceptions. This was also revealed in a longitudinal study of personality and social development (35), in which it was found that how the children experience their parents' behavior is more important than how the parents actually behave. The type of parenting we describe in the present study (i.e., warm and supportive) is called child-centered parenting by Pulkkinen (2017) (35), and based on her 
longitudinal studies, child-centered parenting has been shown to be associated with responsible behavior in children. It is very likely that the high level of physical activity may also be a part of this kind of responsible behavior in children and, thus, could partly explain our results.

The psychosocial home environment and leisure-time physical activity were assessed by self-reported questionnaires, which may be one potential limitation of the present study because self-reports have been shown to have shortcomings with respect to validity and reliability (36). This may not be a concern in terms of the psychosocial home environment, since self-report is the only way to measure perceived home atmosphere and relational tensions. For physical activity, however, more objective measures are available (e.g., using accelerometers). Although these objective measurements do not always correspond well to self-reports, the validity of the physical activity questionnaires we used for leisure-time physical activity has been repeatedly demonstrated (29, 37-39). For the twin analysis, we made the assumptions that neither the twins' psychosocial home environment in childhood and adolescence nor their leisure-time physical activity behavior in young adulthood differ from those of the general population. However, twins are often born prematurely and hence are lower in weight than average singleton newborns (40), but these differences between singletons and twins disappear in adolescence and are not present in adults.

One of the main advantages of the twin study design is that the design can be used to disentangle genetic and environmental effects. A key strength of the present study is also the longitudinal study design, which provides an opportunity to assess the effects of the psychosocial home environment in childhood and adolescence measured at three time-points on leisure-time physical activity behavior in young adulthood. Studying factors over time is not proof of a causal relationship per se, but it enables testing the temporal directions of the association.

A further strength of this study is that both children and parents rated their perceptions of the psychosocial home environment when twins were 12 years old, providing an opportunity to compare the differences between different persons living in the same home environment. Due to the population-based sample, our study sample was relatively heterogeneous in nature with relatively equal sex representation, suggesting good generalizability of the findings. An additional advantage of this study is its large sample size, which ensures sufficient statistical power to detect statistically significant associations. Moreover, various selection biases are unlikely in our study due to the high response and low "lost to followup" rates in the survey. Also, physical activity was assessed as MET indices by taking the frequency, mean duration and mean intensity of leisure-time physical activity sessions into account.

\section{CONCLUSION}

This study demonstrated that the child's subjective view, especially, but also the parents', of having a psychosocial home environment that is warm and supportive in childhood and midadolescence may increase not only the child' subsequent leisure-time physical activity behavior, but also modify additive genetic and unique environmental influences on leisure- 
time physical activity behavior more than 10 years after the influence of the psychosocial home environment in childhood. The interplay between the genes and psychosocial home environment in childhood and adolescence seem to be important when explaining differences in leisure-time physical activity behavior in young adults. For future studies, large randomized controlled trials would help to develop a broader picture of the causal relationships between the childhood and adolescence psychosocial home environment and subsequent leisure-time physical activity behavior. Moreover, replications regarding the modifying role of the childhood and adolescence psychosocial home environment on the genetic influences of leisure-time physical activity are needed. Since our results indicate that leisure-time physical activity is sensitive to the childhood and adolescence home environment, it potentially emphasizes a need to implement more knowledge from social sciences to human genetics to better understand the role of familial effects behind differences in voluntary physical activity behavior. In terms of clinical implications, it would be important to find ways to help parents create a home environment that is both warm and supportive for their children because it can go a long way toward developing their offspring's interest in physical activity even later in life.

\section{Acknowledgments}

The research work has been supported by the Academy of Finland (grant 266592 to S.A. and K.S., grants 100499 , 205585, 141054, 265240, 263278 and 264146 to J.K.), the Finnish Ministry of Education and Culture (to S.A., U.M.K. and K.S.), the Juho Vainio Foundation (to S.A., U.M.K. and K.S.), and the Finnish Cultural Foundation (to S.A.). Data collection of the FinnTwin 12 study has also been supported by the U.S. National Institute of Alcohol Abuse and Alcoholism (grants AA-12502, AA-00145, and AA-09203 to R.J.R.).

\section{References}

1. Aaltonen S, Kujala UM, Kaprio J. Factors behind leisure-time physical activity behavior based on Finnish twin studies: the role of genetic and environmental influences and the role of motives. Biomed Res Int. 2014; 2014:931820.doi: 10.1155/2014/931820. [PubMed: 24809061]

2. de Geus EJ, Bartels M, Kaprio J, Lightfoot JT, Thomis M. Genetics of regular exercise and sedentary behaviors. Twin Res Hum Genet. 2014; 17(4):262-71. [PubMed: 25034445]

3. de Vilhena e Santos DM, Katzmarzyk PT, Seabra AF, Maia JA. Genetics of physical activity and physical inactivity in humans. Behav Genet. 2012; 42(4):559-78. [PubMed: 22426782]

4. Huppertz C, Bartels M, de Zeeuw EL, et al. Individual differences in exercise behavior: stability and change in genetic and environmental determinants from age 7 to 18. Behav Genet. 2016; 46(5):66579. [PubMed: 27406597]

5. Stubbe JH, Boomsma DI, Vink JM, et al. Genetic influences on exercise participation in 37.051 twin pairs from seven countries. PLoS ONE. 2006; 1(1):e22.doi: 10.1371/journal.pone.0000022 [PubMed: 17183649]

6. Aaltonen S, Ortega-Alonso A, Kujala UM, Kaprio J. Genetic and environmental influences on longitudinal changes in leisure-time physical activity from adolescence to young adulthood. Twin Res Hum Genet. 2013; 16(2):535-43. [PubMed: 23449154]

7. Bauman AE, Reis RS, Sallis JF, Wells JC, Loos RJ, Martin BW, Lancet Physical Activity Series Working Group. Correlates of physical activity: why are some people physically active and others not? Lancet. 2012; 380(9838):258-71. [PubMed: 22818938]

8. Maitland C, Stratton G, Foster S, Braham R, Rosenberg M. A place for play? The influence of the home physical environment on children's physical activity and sedentary behaviour. Int J Behav Nutr Phys Act. 2013; 10:99. 5868-10-99. [PubMed: 23958282]

9. Kaushal N, Rhodes RE. The home physical environment and its relationship with physical activity and sedentary behavior: a systematic review. Prev Med. 2014; 67:221-37. [PubMed: 25084562] 
10. Elhakeem A, Cooper R, Bann D, Hardy R. Childhood socioeconomic position and adult leisuretime physical activity: a systematic review. Int J Behav Nutr Phys Act. 2015; 12:92. 015-0250-0. [PubMed: 26138985]

11. Gustafson SL, Rhodes RE. Parental correlates of physical activity in children and early adolescents. Sports Med. 2006; 36(1):79-97. [PubMed: 16445312]

12. Cerin E, Cain KL, Conway TL, et al. Neighborhood environments and objectively measured physical activity in 11 countries. Med Sci Sports Exerc. 2014; 46(12):2253-64. [PubMed: 24781892]

13. Adams MA, Ding D, Sallis JF, et al. Patterns of neighborhood environment attributes related to physical activity across 11 countries: a latent class analysis. Int J Behav Nutr Phys Act. 2013; 10:34. 5868-10-34. doi: 10.1186/1479-5868-10-34 [PubMed: 23497187]

14. Beets MW, Cardinal BJ, Alderman BL. Parental social support and the physical activity-related behaviors of youth: a review. Health Educ Behav. 2010; 37(5):621-44. [PubMed: 20729347]

15. Edwarson CL, Gorely T. Parental influences on different types and intensities of physical activity in youth: a systematic review. Psychol Sport Exerc. 2010; 11(6):522-35.

16. Sallis JF, Prochaska JJ, Taylor WC. A review of correlates of physical activity of children and adolescents. Med Sci Sports Exerc. 2000; 32(5):963-75. [PubMed: 10795788]

17. Van Der Horst K, Paw MJ, Twisk JW, Van Mechelen W. A brief review on correlates of physical activity and sedentariness in youth. Med Sci Sports Exerc. 2007; 39(8):1241-50. [PubMed: 17762356]

18. Biddle SJH, Whitehead SH, O'Donovan TM, Nevill ME. Correlates of participation in physical activity for adolescent girls: A systematic review of recent literature. J Phys Act Health. 2005; 2(4):423-34.

19. Cleland V, Timperio A, Salmon J, Hume C, Telford A, Crawford D. A longitudinal study of the family physical activity environment and physical activity among youth. Am J Health Promot. 2011; 25(3):159-67. [PubMed: 21192744]

20. Crawford D, Cleland V, Timperio A, et al. The longitudinal influence of home and neighbourhood environments on children's body mass index and physical activity over 5 years: the CLAN study. Int J Obes (Lond). 2010; 34(7):1177-87. [PubMed: 20351728]

21. Davison KK, Downs DS, Birch LL. Pathways linking perceived athletic competence and parental support at age 9 years to girls' physical activity at age 11 years. Res Q Exerc Sport. 2006; 77(1): 23-31. [PubMed: 16646349]

22. Dzewaltowski DA, Ryan GJ, Rosenkraz RR. Parental bonding may moderate the relationship between parent physical activity and youth physical activity after school. Psychol Sport Exerc. 2008; 9(6):848-54.

23. Silventoinen K, Volanen SM, Vuoksimaa E, Rose RJ, Suominen S, Kaprio J. A supportive family environment in childhood enhances the level and heritability of sense of coherence in early adulthood. Soc Psychiatry Psychiatr Epidemiol. 2014; 49(12):1951-60. [PubMed: 24619310]

24. Dick DM, Viken R, Purcell S, Kaprio J, Pulkkinen L, Rose RJ. Parental monitoring moderates the importance of genetic and environmental influences on adolescent smoking. J Abnorm Psychol. 2007; 116(1):213-8. [PubMed: 17324032]

25. Latendresse SJ, Rose RJ, Viken RJ, Pulkkinen L, Kaprio J, Dick DM. Examining the etiology of associations between perceived parenting and adolescents' alcohol use: common genetic and/or environmental liabilities? J Stud Alcohol Drugs. 2010; 71(3):313-25. [PubMed: 20409424]

26. Kaprio J. The Finnish twin cohort study: an update. Twin Res Hum Genet. 2013; 16(1):157-62. [PubMed: 23298696]

27. Kaprio J. Twin studies in Finland 2006. Twin Res Hum Genet. 2006; 9(6):772-7. [PubMed: 17254406]

28. Waller K, Kaprio J, Kujala UM. Associations between long-term physical activity, waist circumference and weight gain: a 30-year longitudinal twin study. Int J Obes (Lond). 2008; 32(2): 353-61. [PubMed: 17653065]

29. Leskinen T, Waller K, Mutikainen S, et al. Effects of 32-year leisure time physical activity discordance in twin pairs on health (TWINACTIVE study): aims, design and results for physical fitness. Twin Res Hum Genet. 2009; 12(1):108-17. [PubMed: 19210186] 
30. Williams RL. A note on robust variance estimation for cluster-correlated data. Biometrics. 2000; 56(2):645-6. [PubMed: 10877330]

31. Plomin, R., DeFries, JC., McClearn, GE., McGuffin, P. Behavioral Genetics. New York, United States of America: Worth Publishers; 2000. p. 449

32. Rijsdijk FV, Sham PC. Analytic approaches to twin data using structural equation models. Brief Bioinform. 2002; 3(2):119-33. [PubMed: 12139432]

33. Purcell S. Variance components models for gene-environment interaction in twin analysis. Twin Res. 2002; 5(6):554-71. [PubMed: 12573187]

34. Neale MC, Hunter MD, Pritikin JN, et al. OpenMx 2.0: extended structural equation and statistical modeling. Psychometrika. 2016; 81(2):535-49. [PubMed: 25622929]

35. Pulkkinen, L. Human development from middle childhood to middle adulthood: growing up to be middle-aged (In collaboration with Kokko K). 1st. London: Routledge; 2017. p. 82-83.p. 216-217.p. 309

36. Helmerhorst HJ, Brage S, Warren J, Besson H, Ekelund U. A systematic review of reliability and objective criterion-related validity of physical activity questionnaires. Int J Behav Nutr Phys Act. 2012; 9:103. 5868-9-103. [PubMed: 22938557]

37. Kaprio J, Sarna S, Koskenvuo M, Rantasalo I. The Finnish Twin Registry: formation and compilation, questionnaire study, zygosity determination procedures, and research program. Prog Clin Biol Res. 1978; 24(Pt B):179-84. [PubMed: 569306]

38. Sarna S, Kaprio J, Sistonen P, Koskenvuo M. Diagnosis of twin zygosity by mailed questionnaire. Hum Hered. 1978; 28(4):241-54. [PubMed: 566252]

39. Kujala UM, Kaprio J, Sarna S, Koskenvuo M. Relationship of leisure-time physical activity and mortality: the Finnish twin cohort. JAMA. 1998; 279(6):440-4. [PubMed: 9466636]

40. Buckler JM, Green M. A comparison of the early growth of twins and singletons. Ann Hum Biol. 2004; 31(3):311-32. [PubMed: 15204347] 


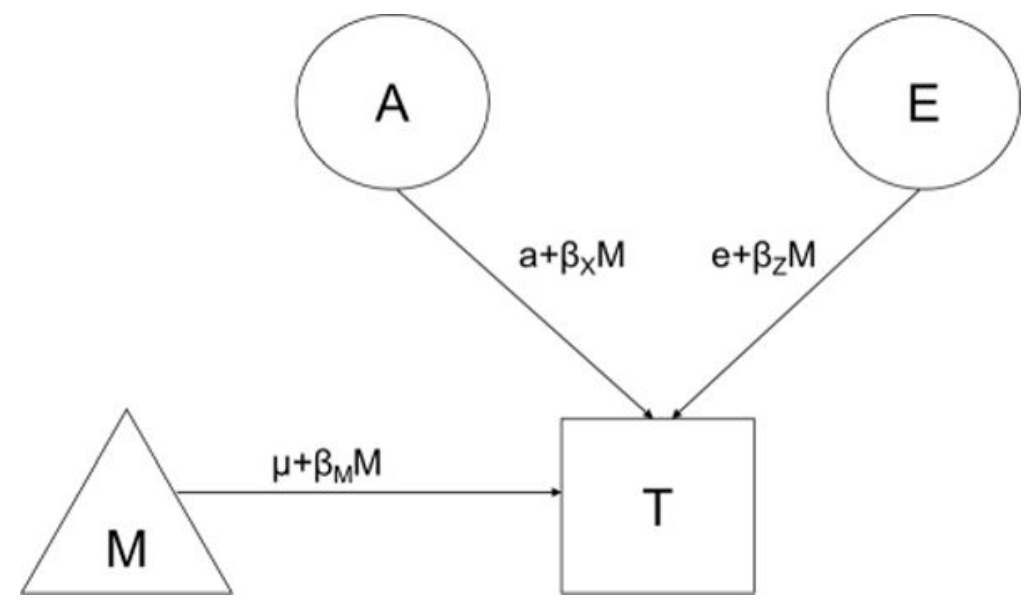

Figure 1.

A path diagram of the gene-environment interaction model. A=additive genetic variation, $\mathrm{E}=$ unique environmental variation, $\mathrm{M}=$ environmental moderator, $\mu=$ mean path coefficient, $\mathrm{a}=$ additive genetic path coefficient, e=unique environmental path coefficient, $\beta_{\mathrm{M}} \mathrm{M}=\mathrm{mean}$ moderator effect, $\beta_{\mathrm{A}} \mathrm{M}=$ additive genetic moderator effect, $\beta_{\mathrm{E}} \mathrm{M}=$ unique environment moderator effect. 


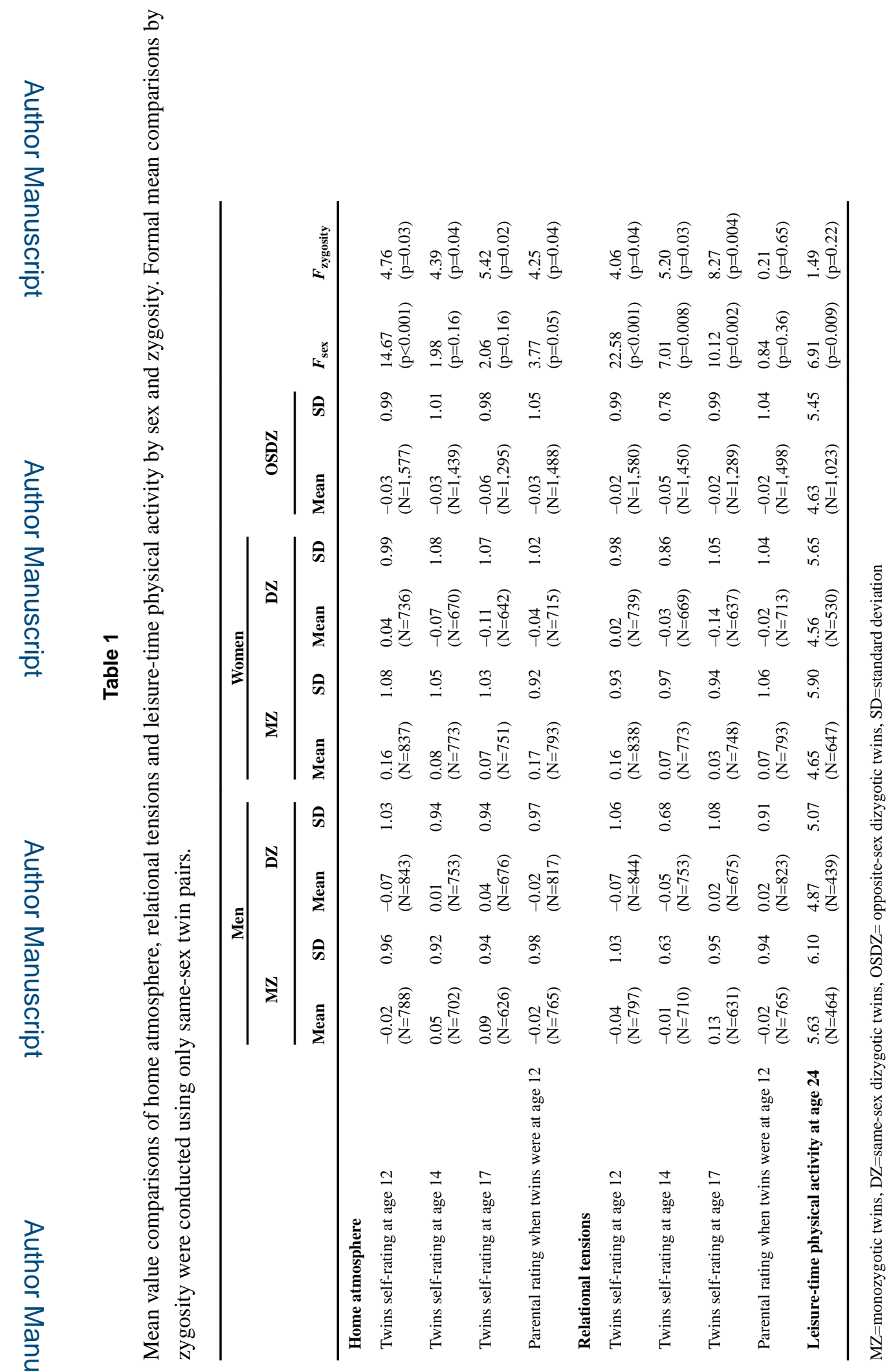

Med Sci Sports Exerc. Author manuscript; available in PMC 2019 April 01. 


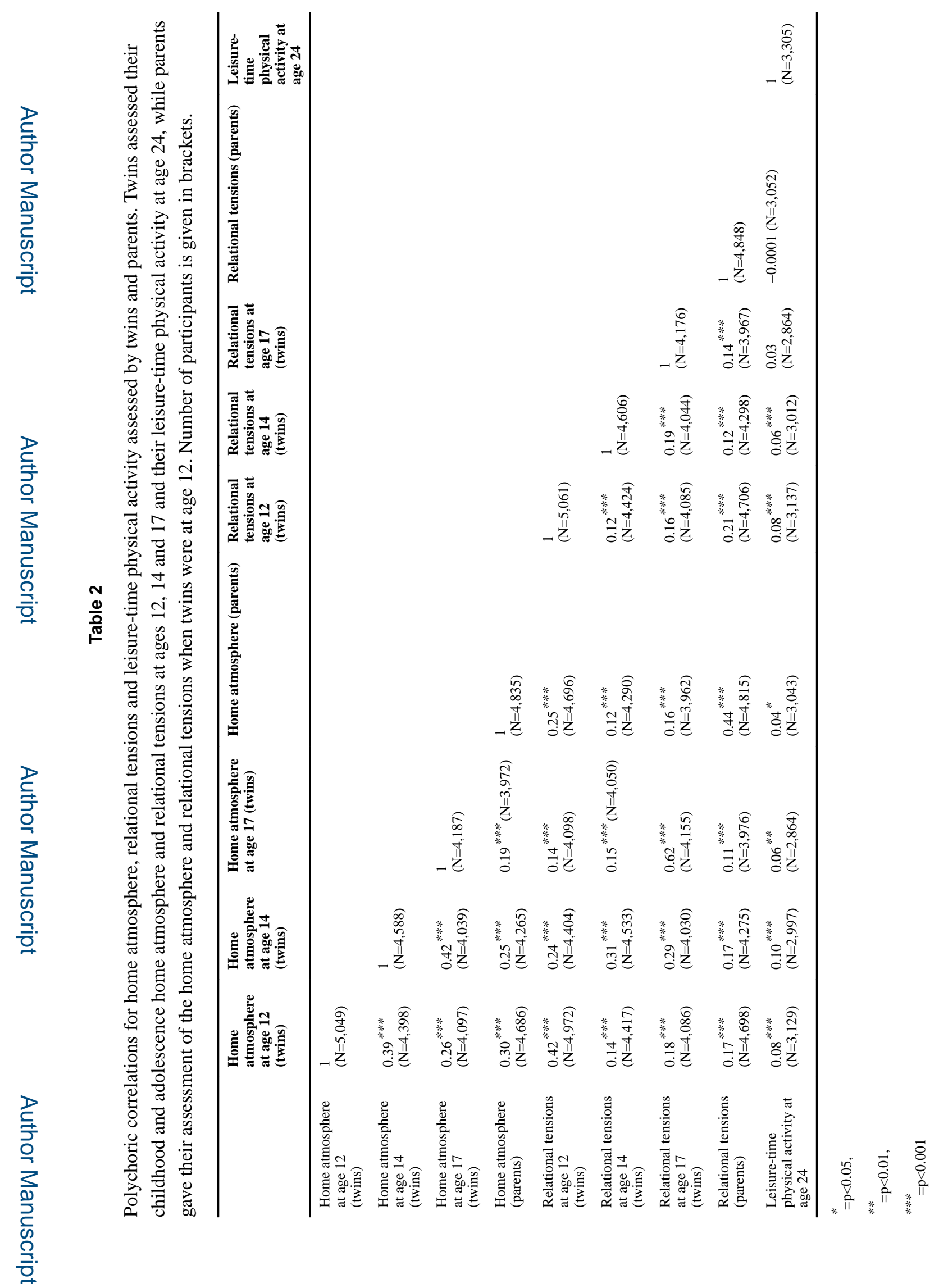

Med Sci Sports Exerc. Author manuscript; available in PMC 2019 April 01. 


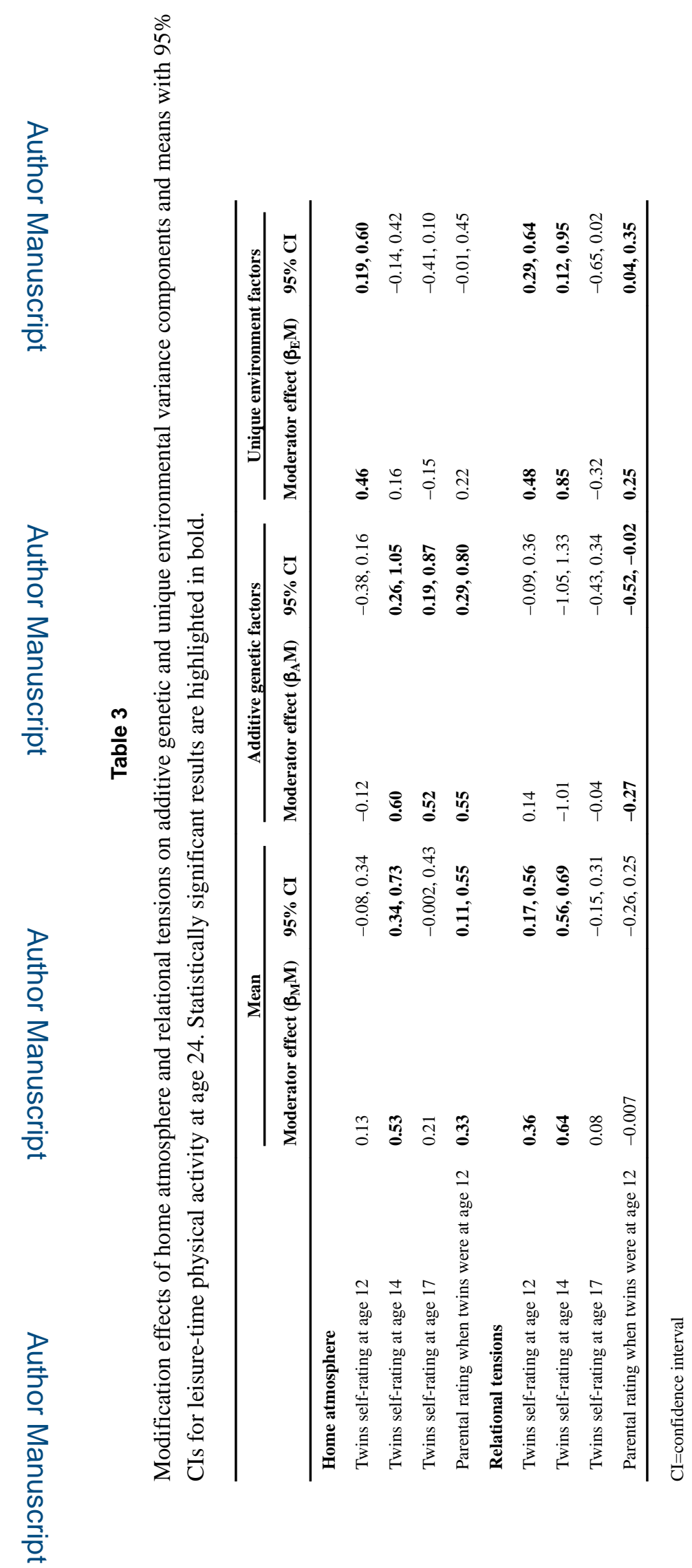

Med Sci Sports Exerc. Author manuscript; available in PMC 2019 April 01. 